Sektion 10

\section{Pathophysiologie und Aerosolmedizin}

Nachfolgend berichten wir über die Tätigkeiten der Sektion 10 für den Zeitraum März 2016 bis März 2017.

\section{Die Angaben der Sektion 10 auf der Homepage der DGP wurden 2016 überarbeitet und aktualisiert}

Neben klassischen Themen wie Lungenfunktionsanalyse treibt die Sektion 10 die Diagnostik von Entzündungs- und Umbauprozessen im Atemtrakt voran. Ein wissenschaftlicher Schwerpunkt ist zudem die Weiterentwicklung von Exhalatdiagnostik einschließlich lonenbeweglichkeitsspektrometrie. Die Sektion 10 widmet sich auch dem Atemwegskondensat und Methoden, die unter dem Oberbegriff der „elektronischen Nase“ subsumiert werden können. Mit dieser innovativen Technik kann in der Atemluft eine Vielzahl von Substanzen nachgewiesen werden - und das in geringsten Konzentrationen.

Auf dem Gebiet der Aerosolmedizin, die Spezialwissen erfordert, fungiert die Sektion 10 als kompetenter Ansprechpartner in allen Fragen der Inhalationstechnologie und unterstützt Schulungsmaßnahmen. Das Erarbeiten pathophysiologischer Konzepte für die Indikationsstellung einer Sauerstofftherapie hat die Sektion sich ebenfalls zur Aufgabe gemacht. Gerade die Pathophysiologie ist ein wichtiges Bindeglied zu den anderen wissenschaftlichen Sektionen der DGP und ermöglicht viele sinnvolle und notwendige Kooperationen.

Zahlreiche Symposien und Vorträge auf den DGP-Kongressen zeugen von der produktiven Tätigkeit der interdisziplinären Sektion. Dieser Erfolg soll auch in Zukunft fortgesetzt werden.

\section{DGP-Kongress in Leipzig,}

\section{2. bis 5. März 2016}

Im Rahmen der Jahrestagung der DGP 2016 in Leipzig fanden unter Federführung der Sektion Pathophysiologie und Aerosolmedizin vier klinische Symposien, zwei Postgraduiertenkurse (Grund- lagen der Lungenfunktionsdiagnostik, Praktische Inhalationstherapie bei Asthma und COPD) sowie der traditionelle Workshop des Arbeitskreises „Respiratorisches System“ der DVG statt. Auch eine Veranstaltung mit freien Vorträgen konnte erfolgreich durchgeführt werden. Zudem war die Sektion thematisch an mehreren Veranstaltungen der kooperierenden Sektionen beteiligt.

\section{Herbsttagung 2016}

Am 11. und 12. November 2016 fand die Herbsttagung der Sektion Pathophysiologie und Aerosolmedizin in Zusammenarbeit mit der Sektion 4 (Infektiologie und Tuberkulose) und der Sektion 14 (Zellbiologie) in Hannover mit ca. 60 Teilnehmern statt. Angesichts des mangelnden Nachwuchses in der Sektion 10 wird 2017 keine durch die Sektion 10 organisierte Herbsttagung stattfinden. Stattdessen soll die Nachwuchsförderung verstärkt forciert werden.

\section{Deutsches Aerosol Therapie Seminar}

Die Inhalationstherapie entwickelt sich in rasantem Tempo. Am 4. und 5. November 2016 fand zum 19. Mal das Deutsche Aerosol Therapie Seminar in Waldeck am Edersee statt. Teilnehmer aus unterschiedlichen Disziplinen (Medizin, Physik, Biologie, Pharmazie) trafen sich hier, um über Fortschritte, Chancen und Grenzen der Aerosoltherapie zu diskutieren.

\section{Erster Marburger Rehatag}

Am 3.12.2016 fand zusammen mit der Sektion 12 der erste Marburger Rehatag statt. Das Konzept soll im Jahr 2017 verstetigt werden. Der 2. Marburger Rehatag ist für den 11.11.2017 terminiert.

\section{Nachwuchsarbeit}

Unter der Ägide von Felix Herth und Christoph Lange erfolgte die Durchführung der Winterakademie im Pitztal vom 8. bis 13.1.2017. Vom 2. bis 7.7. 2017 wird unter Leitung von Rembert Koczulla, Maren Schuhmann und Matthias Held die erste Sommerakademie zur Nachwuchsförderung in Berchtesgaden, Schönau am Königssee, stattfinden.

\section{Neuwahl des Sprechers sowie des} Stellvertreters der Sektion 10

Auf der diesjährigen Sektionssitzung im Rahmen des DGP-Kongresses in Stuttgart erfolgte die turnusmäßige Neuwahl des Sprechers sowie des Stellvertreters der Sektion 10 für die nächsten 2 Jahre. Unter Moderation von Herrn Prof. Koczulla wurden jeweils bei 0 Gegenstimmen und jeweils 1 Enthaltung als Sprecherin Frau Dr. Verena Knipel aus Köln sowie als Vertreterin Frau Dr. Ulrike Olgemöller aus Göttingen gewählt.

\section{Publikationen}

Auch im vergangenen Jahr wurden zahlreiche Publikationen von Sektionsmitgliedern in diversen Fachzeitschriften publiziert:

\section{Empfehlungen}

Respiratory Muscle Training: State of the Art. Göhl O, Walker DJ, Walterspacher S, Langer D, Spengler CM, Wanke T, Petrovic $M$, Zwick RH, Stieglitz S, Glöckl R, Dellweg D, Kabitz HJ. Pneumologie 2016; 70: $37-48$

\section{Publikationen in Pneumologie}

Checklists for the Assessment of Correct Inhalation Therapy. Knipel V, Schwarz S, Magnet FS, Storre JH, Criée CP, Windisch W; German Airway League. Pneumologie 2017; 71: $96-105$

Diagnosis and Treatment of Diaphragmatic Dysfunction. Windisch W, Schönhofer B, Magnet FS, Stoelben E, Kabitz HJ. Pneumologie. 2016; 70: 454-461

Expert Meeting on COPD: Personalized Treatment of COPD - Wishful Thinking or Reality? Lorenz J, Bals R, Ewert R, Jany B, Koczulla AR, Köhnlein T, Randerath WJ, Steinkamp G, Watz H, Welte T. Pneumologie. 2016; 70: 638-650

\section{Weitere ausgewählte wichtige Publikationen}

Inhalation device requirements for patients' inhalation maneuvers. Haidl $\mathrm{P}$, Heindl S, Siemon K, Bernacka M, Cloes RM. Respir Med 2016; 118: 65 - 75

A dual center study to compare breath volatile organic compounds from smokers and non-smokers with and without 
COPD Gaida A, Holz O, Nell C, Schuchardt S, Lavae-Mokhtari B, Kruse L, Boas U, Langejuergen J, Allers M, Zimmermann S, Vogelmeier C, Koczulla AR, Hohlfeld JM. J Breath Res 2016; 10: 026006

\section{Therapie mit Aerosolen}

Atemwegs- und Lungenkrankheiten, Jahrgang 42, Nr. 10/2016 - Dustri Verlag:

- Grundlagen der Aerosoltherapie,

S. Canisius und G. Scheuch.

- Treibgasbetriebene Dosieraerosole, der Respimat ${ }^{\circledR}$ und die Spacer-Systeme, Th. Voshaar

- Trockenpulverinhalationssysteme, V. Knipel

- Inhalationstherapie mit Verneblern, P. Haidl

- Schulung und typische Anwendungsprobleme in der Praxis, B. Esche

- Auswahlkriterien, Schulung, Zufriedenheit, Adhärenz und klinische Effekte bei der Inhalationstherapie, Th. Voshaar

- Inhalationstherapie auf Intensivstation und im Notfall, P. Haidl und D. Dellweg

\section{Ausblick für das Jahr 2017}

Im bevorstehenden Jahr sind Aktivitäten der Sektion 10 bei diversen Veranstaltungen geplant. Unter anderem wird vom 2. bis 7. Juli die Sommer-Akademie der DGP in Schönau am Königssee ausgerichtet. Motivierten Kolleginnen und Kollegen in der Weiterbildung sowie Studenten, die sich für das Fach Pneumologie interessieren, soll hier die Möglichkeit geboten werden, sich in dem Fach Pneumologie weiterzuentwickeln.

Für den 11.11.2017 ist die Ausrichtung des 2. Marburger Rehatages in Zusammenarbeit mit der Sektion 12 Rehabilitation, Prävention und Tabakkontrolle, geplant.

Insgesamt soll 2017 die wissenschaftliche Präsenz gestärkt und der Nachwuchs gefördert werden. Alle Mitglieder der Sektion 10 sowie Interessierte auf dem Gebiet Pathophysiologie und Aerosolmedizin werden daher aufgerufen, hierzu beizutragen.

Dr. Verena Knipel, Köln und

Dr. Ulrike Olgemöller, Göttingen 